\begin{tabular}{|c|c|}
\hline $\begin{array}{l}\text { AUG } 20199621 \text { engineERING DATA TRANSMITTAL } \\
\text { sta, } 15\end{array}$ & 1. EDT NO 614552 \\
\hline
\end{tabular}



BD-7400-172-2 (04/94) GEF097 


\title{
Releases From Failed HEPA Filters Due to an Overpressurization Event
}

\author{
G. W. Ryan \\ Westinghouse Hanford Company, Richland, WA 99352 \\ U.S. Department of Energy Contract DE-AC06-87RL10930

\begin{tabular}{|c|c|c|}
\hline $\begin{array}{l}\text { DT/ECN: } \\
\text { rg Code: } \\
\text { \&R Code: }\end{array}$ & $\begin{array}{l}614552 \\
8 M 100 \\
\text { EW3120071 }\end{array}$ & $\begin{array}{l}\text { UC: } 510 \\
\text { Charge Code: } \\
\text { Total Pages: }\end{array}$ \\
\hline
\end{tabular}

Key Words: HEPA filter blow out, pressurization, radioactive materials, tank farms, TWRS, ventilation system

Abstract: This document supports the development and presentation of the following accident scenario in the TWRS Final Safety Analysis Report:

HEPA Filter Failure - Exposure to High Temperature or Pressure.

The calculations needed to quantify the risk associated with this accident scenario are included within.

TRADEMARK DISCLAIMER. Reference herein to any specific comercial product, process, or service by trade name, trademark, manufacturer, or otherwise, does not necessarily constitute or imply its endorsement, recommendation, or favoring by the United States Goverment or any agency thereof or its contractors or subcontractors.

Printed in the United States of America. To obtain copies of this document, contact: HHC/BCS Document Control Services, P.O. Box 1970, Mailstop H6-08, Richland WA 99352, Phone (509) 372-2420; Fax (509) 376-4989.

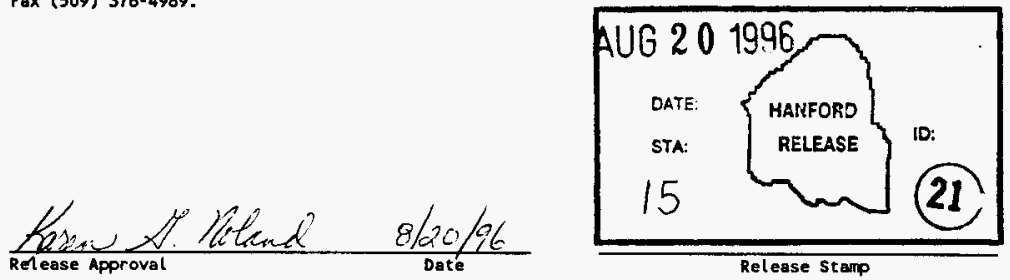

Approved for Public Release 
RELEASES FROM FAILED HEPA FILTERS DUE TO AN OVERPRESSURIZATION EVENT REVISION 0

Tank Waste Remediation System Final Safety Analysis Report Project Safety Analysis \& Nuclear Engineering

NOTE: ACCORDING TO SECTION WP-6.7, REV. O OF WHC-CM-6-32, SAFETY ANALYSIS AND NUCLEAR ENGINEERING WORK PROCEDURES, CALCULATION NOTES ARE USED TO DOCUMENT THE ORIGINATOR'S ANALYSIS BUT ARE NOT TO BE USED TO AUTHORIZE ACTIVITIES OR JUSTIFY FACILITY MODIFICATIONS, OR CHANGES TO AN AUTHORIZATION BASIS, SAFETY BASIS, OR DESIGN BASIS.

August 1996 
WHC-SD-WM-CN-063 REV 0

This page intentionally left blank. 


\section{CONTENTS}

1.0 INTRODUCTION AND PURPOSE ................ 7 of 26

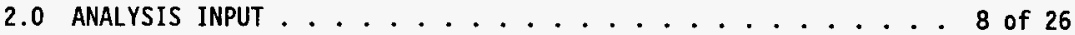

3.0 ASSUMPTIONS ................................... 26

4.0 ANALYTICAL METHODS ............................. of 26

5.0 RADIOLOGICAL DOSE CONSEQUENCE ANALYSIS . . . . . . . . 12 of 26

6.0 TOXICOLOGICAL EXPOSURE CONSEQUENCE ANALYSIS . . . . . . . 15 of 26

7.0 ANALYSIS RESULTS ............................ of 26

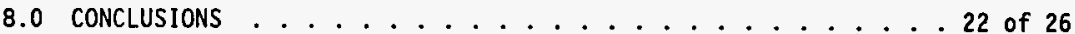

9.0 REFERENCES . . . . . . . . . . . . . . . . . 22 of 26

APPENDICES

A PEER REVIEW AND HEDOP REVIEW CHECKLISTS . . . . . . . 23 of 26 


\section{LIST OF TABLES}

1. HEPA Filter Release Amounts (Q) from Various TWRS Ventilation Systems .............................. 8 of 26

2. Unit Liter Doses Used in This Analysis ........... 9 of 26

3. Unmitigated Radiological Dose Consequences from Continuous, Unfiltered Releases................. 10 of 26

4. Unmitigated Toxicological Exposure Consequences from Continuous, Unfiltered Releases (from WHC-SD-WM-CN-062, Rev. 0) ... 11 of 26

5. Total Unmitigated Radiological Dose Consequences from a Pressurized HEPA Release Event and a Continuous, Unfiltered ReTease . . . 18 of 26

6. Total Unmitigated Toxicological Exposure Consequences from a Pressurized HEPA Release Event and a Continuous, Unfiltered Release ................................... 20 of 26

7. Risk Guidelines for Radiological Dose and Toxicological Exposure Consequences ................................ 21 of 


\section{LIST OF TERMS}

$\begin{array}{ll}\text { AWF } & \text { aging waste facility } \\ \text { DST } & \text { double-shell tank } \\ \text { FSAR } & \text { final safety analysis report } \\ \text { HEDOP } & \text { Hanford Environmental Dose Overview Panel } \\ \text { HEPA } & \text { high efficiency particulate air (filter) } \\ \text { PF } & \text { partition fraction } \\ \text { rem } & \text { radiation effective man } \\ \text { RF } & \text { respirable fraction } \\ \text { SST } & \text { single-shell tank } \\ \text { SV } & \text { sievert } \\ \text { TWRS } & \text { Tank Waste Remediation System } \\ \text { ULD } & \text { unit liter dose } \\ \text { WHC } & \text { Westinghouse Hanford Company }\end{array}$


WHC-SD-WM-CN-063 REV 0

This page intentionally left blank.

6 of 26 


\section{RELEASES FROM FAILED HEPA FILTERS DUE TO AN OVERPRESSURIZATION EVENT}

\subsection{INTRODUCTION AND PURPOSE}

This document supports the development and presentation of the following accident scenario in the TWRS Final Safety Analysis Report (FSAR):

\section{HEPA Filter Failure - Exposure to High Temperature or Pressure.}

Passive or active ventilation is maintained across single-shell and double-shell waste tanks, double-contained receiver tanks (DCRTs), the 204-AR Waste Unloading Facility, the 244-AR Receiver Vault, the compactor building, and catch tanks 241-A-417 and 241-AX-152.

\section{ACCIDENT SCENARIO DEVELOPNENT AND DESCRIPTION}

It is postulated that a pressure differential as little as $0.63 \mathrm{lb} / \mathrm{in}^{2}$ (10 inches water gauge) will fail HEPA filters in a ventilation system (either active or passive) and create a sudden, unfiltered release of radiological and toxicological contaminants. Subsequent to the HEPA filter release, a continuous, unfiltered release of headspace air is assumed to occur. Doses associated with the continuous, unfiltered release have been calculated in WHC-SD-WM-CN-062, Rev. 0, and are considered appropriate to be added to the results of the analysis for the initial HEPA filter release as presented in this calculation note.

Unlike the evaluation performed in WHC-SD-WM-CN-062, HEPA Filter Fire (and Subsequent Unfiltered Release), a headspace pressurization cannot occur independent of an additional accident initiator. There are many initiators that could lead to a pressurization resulting in a HEPA filter release. These include steam intrusions, an in-tank fuel fire/deflagration, or an organic salt-nitrate reaction. These accident scenarios are evaluated separately (and include where appropriate, the dose associated with a HEPA filter breach and the continuous, unfiltered release following the breach) for the TWRS FSAR.

The intent of this document is to provide the radiological dose and toxicological exposure consequences associated with a HEPA filter rupture by pressurization and the subsequent unfiltered, continuous release following the pressurization.

\section{ACCIDENT FREQUENCY DEYELOPMENT}

Since the frequency associated with a specific accident scenario may vary, this document presents the radiological dose for comparison with all accident frequencies (anticipated, unlikely, and extremely unlikely). Since toxicological exposure consequences are calculated differently, only the values for an anticipated accident frequency are presented here. Using the method presented in this document, a user can calculate similar exposure consequences for the other accident frequencies (unlikely and extremely unlikely). The radiological risk acceptance guidelines are referenced from WHC-CM-4-46, Rev. 1, whereas the toxicological risk acceptance guidelines are built-in to the calculational methodology of WHC-SD-WM-SARR-011. 


\subsection{ANALYSIS INPUT}

\section{Amount of Material Released (Q)}

The material release amounts for a rapid pressurization from the various ventilation systems are calculated in WHC-SD-WM-CN-054, Waste Tank Ventilation System Waste Material Accumulations, and summarized in Table 1. The release amounts from a contaminated filter are calculated using a.release fraction of $1 \times 10^{-2}$. This conservative release fraction is given in Section 5.4.4.2 of DOE-HDBK-3010-94 and corresponds to the material that would be released from crushing a filter in an unconfined space.

Table 1. HEPA Filter Release Amounts (Q) from Various TWRS Ventilation Systems.

\begin{tabular}{|c|c|c|}
\hline Facility/Vent System & Type of Waste Released & Amount Released (L) \\
\hline $\begin{array}{l}241-S X / 241-C-106 \\
\text { (SSTs with active } \\
\text { ventilation) }\end{array}$ & SST Solids & $6.13 \mathrm{E}-03$ \\
\hline $\begin{array}{l}\text { SSTs with Passive } \\
\text { Breather Filters } \\
\text { (SSTs with Passive } \\
\text { Ventilation } 1 \text { and 2) }\end{array}$ & $\begin{array}{l}\text { SST Solids } \\
(24 \times 24 \times 12 \text { Filter }) \\
\text { SST Solids } \\
(12 \times 12 \times 12 \text { Filter })\end{array}$ & $\begin{array}{l}1.27 \text { E-04 } \\
4.72 \text { E-05 }\end{array}$ \\
\hline DCRTs & SST Liquids & $2.28 \mathrm{E}-03$ \\
\hline $\begin{array}{l}241-S Y / 241-A N / 241-A P \\
241-A W \text { (DSTs) }\end{array}$ & DST Liquids & $9.79 \mathrm{E}-04$ \\
\hline $\begin{array}{l}\text { 241-AY/241-AZ (DSTs } \\
\text { using AWF Liquid) }\end{array}$ & AWF Liquids & 5.62 E-03 \\
\hline $\begin{array}{l}\text { 204-AR Waste Unloading } \\
\text { Facility }\end{array}$ & DST Liquids & 2.37 E-03 \\
\hline 244-AR Receiver Vault & DST Liquids & $2.37 \mathrm{E}-03$ \\
\hline Compactor Building & DST Liquids & $9.79 E-04$ \\
\hline Catch Tanks & DST Liquids & $9.79 E-04$ \\
\hline
\end{tabular}




\section{Unit Liter Doses (ULDs)}

The ULDs for this analysis are taken from WHC-SD-WM-SARR-037, Rev. 0 , Deve7opment of Radiological Concentrations and Unit Liter Doses for TWRS FSAR Radiological Consequence Calculations. Table 2 provides the ULD's used in this analysis:

Table 2. Unit Liter Doses Used in This Analysis.

\begin{tabular}{|l|c|c|}
\hline Composite & $\begin{array}{c}\text { Inhaiation ULD } \\
(\mathrm{Sv} / \mathrm{L})\end{array}$ & $\begin{array}{c}\text { Ingestion ULD } \\
\left(\mathrm{Sv}-\mathrm{m}^{3} / \mathrm{s}-\mathrm{L}\right)\end{array}$ \\
\hline SST Liquids & $1.1 \mathrm{E}+04$ & 0.052 \\
\hline SST Solids & $2.2 \mathrm{E}+05$ & 4.1 \\
\hline DST Liquids & $6.1 \mathrm{E}+03$ & 0.068 \\
\hline AWF Liquids & $1.4 \mathrm{E}+03$ & 0.092 \\
\hline
\end{tabular}

The use of these ULDs is consistent with the calculations performed in WHC-SD-WM-CN-062.

3. Dispersion Coefficients ( $\left.X / Q^{\prime} s\right)$

Onsite - $\quad 3.41 \times 10^{-2} \mathrm{~s} / \mathrm{m}^{3}$ (WHC-SD-WM-SARR-016).
Offsite - $2.83 \times 10^{-5} \mathrm{~s} / \mathrm{m}^{3}$ (WHC-SD-WM-SARR-016).

\section{Breathing Rate (R)}

$3.3 \times 10^{-4} \mathrm{~m}^{3} / \mathrm{s}$ light activity breathing rate is used to calculate consequences to both the onsite and offsite receptors (WHC-SD-WM-SARR-016).

\section{Unmitigated Continuous, Unfiltered Radiological Dose and Toxicological Exposure Consequences}

In WHC-SD-WM-CN-062, Rev. 0, the unfiltered release was calculated for 12 hours (onsite), 24 hours (offsite) and a year (onsite and offsite). For the one year unfiltered release to the onsite receptor, the dose is adjusted by an occupancy factor of 0.286 to account for the actual time spent at work. A partition fraction (PF) of 1.0 E-10 was used for SSTs with active and passive ventilation, DCRTs, the 204-AR, 244-AR, and a partition fraction of 1.0 E-08 was used for the DST tanks (including AWF tanks). It was assumed that the Compactor Building and Catch Tanks have no liquid therefore, there is no unfiltered release from these facilities. The unmitigated radiological doses and toxicological exposures associated with an unmitigated continuous, unfiltered vent system release calculated in WHC-SD-WM-CN-062 are summarized in Tables 3 and 4 , respectively. The values is Tables 3 and 4 do not include the amount released from the burned HEPA filter (WHC-SD-WM-CN-062). 
Table 3. Unmitigated Radiological Dose Consequences from Continuous, Unfiltered Releases (from WHC-SD-WM-CN-062, Rev. 0 ).

\begin{tabular}{|l|c|c|c|c|}
\hline & \multicolumn{2}{|c|}{$\begin{array}{c}\text { Onsite Dose } \\
\text { Consequences (SV) }\end{array}$} & \multicolumn{2}{c|}{$\begin{array}{c}\text { Offsite Dose } \\
\text { Consequences (Sv) }\end{array}$} \\
\hline Facility/Vent System & $12 \mathrm{hr}$ & $\begin{array}{c}\text { Annual } \\
(8760 \mathrm{hr})\end{array}$ & $24 \mathrm{hr}$ & $\begin{array}{c}\text { Annual } \\
(8760 \mathrm{hr})\end{array}$ \\
\hline $\begin{array}{l}241-S X / 241-C-106 \\
\text { (SSTs with active } \\
\text { ventilation) }\end{array}$ & $1.37 \mathrm{E}-02$ & $7.12 \mathrm{E}-02$ & $1.02 \mathrm{E}-05$ & $8.19 \mathrm{E}-05$ \\
\hline $\begin{array}{l}\text { SSTs with Passive } \\
\text { Breather Filters (SSTs } \\
\text { with Passive } \\
\text { Ventilation 1 and 2) }\end{array}$ & $2.82 \mathrm{E}-06$ & $1.47 \mathrm{E}-05$ & $2.11 \mathrm{E}-09$ & $1.69 \mathrm{E}-08$ \\
\hline DCRTs & $4.10 \mathrm{E}-04$ & $2.14 \mathrm{E}-03$ & $2.93 \mathrm{E}-07$ & $2.34 \mathrm{E}-06$ \\
\hline $\begin{array}{l}\text { 241-SY/241-AN/241-AP } \\
\text { 241-AW (DSTs) }\end{array}$ & $6.31 \mathrm{E}-03$ & $3.29 \mathrm{E}-02$ & $4.61 \mathrm{E}-06$ & $3.69 \mathrm{E}-05$ \\
\hline $\begin{array}{l}\text { 241-AY/241-AZ (DSTs } \\
\text { using AWF Liquid) }\end{array}$ & $4.98 \mathrm{E}-03$ & $2.60 \mathrm{E}-02$ & $4.34 \mathrm{E}-06$ & $3.47 \mathrm{E}-05$ \\
\hline $\begin{array}{l}\text { 204-AR Waste Unloading } \\
\text { Facility }\end{array}$ & $1.08 \mathrm{E}-04$ & $5.65 \mathrm{E}-04$ & $7.92 \mathrm{E}-08$ & $6.33 \mathrm{E}-07$ \\
\hline 244-AR Receiver Vault & $8.13 \mathrm{E}-04$ & $4.24 \mathrm{E}-01$ & $5.94 \mathrm{E}-07$ & $4.75 \mathrm{E}-06$ \\
\hline Compactor Building & N/A & N/A & N/A & N/A \\
\hline Catch Tanks & N/A & N/A & N/A & N/A \\
\hline
\end{tabular}


Table 4. Unmitigated Toxicological Exposure Consequences from Continuous, Unfiltered Releases (from WHC-SD-WM-CN-062, Rev. 0).

\begin{tabular}{|c|c|c|}
\hline Facility/Vent System & $\begin{array}{c}\text { Onsite } \\
\text { Exposure } \\
\text { Consequences } \\
\end{array}$ & $\begin{array}{c}\text { Offsite } \\
\text { Exposure } \\
\text { Consequences } \\
\end{array}$ \\
\hline $\begin{array}{l}241-S X / 241-C-106 \\
\text { (SSTs with active } \\
\text { ventilation) }\end{array}$ & $1.32 \mathrm{E}-02$ & $3.10 \mathrm{E}-05$ \\
\hline $\begin{array}{l}\text { SSTs with Passive } \\
\text { Breather Filters (SSTs } \\
\text { with Passive } \\
\text { Ventilation } 1 \text { and 2) }\end{array}$ & $1.43 \quad E-07$ & 2.24 E-09 \\
\hline DCRTs & $1.90 \quad E-03$ & $1.58 \mathrm{E}-06$ \\
\hline $\begin{array}{l}241-S Y / 241-A N / 241-A P \\
241-A W \text { (DSTs) }\end{array}$ & $5.28 E-02$ & $4.40 \mathrm{E}-05$ \\
\hline $\begin{array}{l}\text { 241-AY/241-AZ (DSTs } \\
\text { using AWF Liquid) }\end{array}$ & $1.89 \mathrm{E}-01$ & $1.59 \mathrm{E}-04$ \\
\hline $\begin{array}{l}\text { 204-AR Waste Unloading } \\
\text { Facility }\end{array}$ & $9.44 \mathrm{E}-04$ & $7.93 \mathrm{E}-07$ \\
\hline 244-AR Receiver Vault & 6.80 E-03 & $5.66 \mathrm{E}-06$ \\
\hline Compactor Building & $N / A$ & $N / A$ \\
\hline Catch Tanks & $N / A$ & $N / A$ \\
\hline
\end{tabular}

- Unmitigated continuous toxicological exposure consequences are calculated using a $\mathrm{L} / \mathrm{s}$ release rate and the final calculation is not duration sensitive for this accident evaluation.

\subsection{ASSUMPTIONS}

No assumptions have been made in this analysis.

\subsection{ANALYTICAL METHODS}

The methodology that is used to calculate radiological dose consequences is documented in WHC-SD-WM-SARR-016, Rev. 2, Tank Waste Compositions and Atmospheric Dispersion Coefficients for use in ASA Consequence Assessments and WHC-SD-WM-SARR-037, Rev. 0, Development of Radiological Concentrations and Unit Liter Doses for TWRS FSAR Radiological Consequence Calculations.

The methodology that is used to calculate toxicological exposure consequences is documented in WHC-SD-WM-SARR-011, Rev. 2, Toxic Chemical Considerations for Tank Farm Releases. 


\subsection{RADIOLOGICAL DOSE CONSEQUENCE ANALYSIS}

Using the formula presented on page 4-4 of WHC-SD-WM-SARR-016, Rev. 2 and modifications for a 24 hour ingestion dose to the offsite receptor from WHC-SD-WM-SARR-037, Rev. 0, the radiological dose consequences can be calculated.

Onsite Consequences:

$$
D(S v)=Q(L) \times \frac{X}{Q^{\prime}}\left(s / m^{3}\right) \times R\left[\mathrm{~m}^{3} / \mathrm{s}\right] \times \mathrm{ULD}_{H}[\mathrm{~Sv} / \mathrm{L}]
$$

SSTs with Active Ventilation -
$D(S v)=$
$\left(6.13 \times 10^{-3} \mathrm{~L}\right)$
L) $\left(3.41 \times 10^{-2} \mathrm{~s} / \mathrm{m}^{3}\right)$
$\left(3.3 \times 10^{-4} \mathrm{~m}^{3} / \mathrm{s}\right)\left(2.2 \times 10^{5} \mathrm{~Sv} / \mathrm{L}\right)$
$D(S v)=$
$1.5 \times 10^{-2} \mathrm{~Sv}$

SSTs with Passive Ventilation 1 -
D $(S v)=$
$\left(1.27 \times 10^{-4} \mathrm{~L}\right)$
L) $\left(3.41 \times 10^{-2} \mathrm{~s} / \mathrm{m}^{3}\right)\left(3.3 \times 10^{-4} \mathrm{~m}^{3} / \mathrm{s}\right)$
$\left(2.2 \times 10^{5} \mathrm{SV} / \mathrm{L}\right)$
$D(S v)=$
$3.1 \times 10^{-4} \mathrm{SV}$

SSTs with Passive Ventilation 2 -
$D(S v)=\left(4.72 \times 10^{-5} \mathrm{~L}\right)\left(3.41 \times 10^{-2} \mathrm{~s} / \mathrm{m}^{3}\right)\left(3.3 \times 10^{-4} \mathrm{~m}^{3} / \mathrm{s}\right)\left(2.2 \times 10^{5} \mathrm{~Sv} / \mathrm{L}\right)$
$D(S V)=1.2 \times 10^{-4} \mathrm{SV}$
DCRTs -
$D(\mathrm{~Sv})=\left(2.28 \times 10^{-3} \mathrm{~L}\right)\left(3.41 \times 10^{-2} \mathrm{~s} / \mathrm{m}^{3}\right)\left(3.3 \times 10^{-4} \mathrm{~m}^{3} / \mathrm{s}\right)\left(1.1 \times 10^{4} \mathrm{~Sv} / \mathrm{L}\right)$
$D(S v)=2.8 \times 10^{-4} \mathrm{SV}$
OSTS -
$D(S v)=\left(9.79 \times 10^{-4} \mathrm{~L}\right)\left(3.41 \times 10^{-2} \mathrm{~s} / \mathrm{m}^{3}\right)\left(3.3 \times 10^{-4} \mathrm{~m}^{3} / \mathrm{s}\right)\left(6.1 \times 10^{3} \mathrm{~Sv} / \mathrm{L}\right)$
$D(S v)=6.7 \times 10^{-5} \mathrm{~Sv}$

DSTs (using AWF Liquids) -
D $(\mathrm{SV})=$
$\left(5.62 \times 10^{-3} \mathrm{~L}\right)\left(3.41 \times 10^{-2} \mathrm{~s} / \mathrm{m}^{3}\right)$
$\left(3.3 \times 10^{-4} \mathrm{~m}^{3} / \mathrm{s}\right)\left(1.4 \times 10^{3} \mathrm{~Sv} / \mathrm{L}\right)$
$D(S v)=$
$8.9 \times 10^{-5} \mathrm{~Sv}$ 
204-AR Waste Unioading Facility -

$D(\mathrm{~Sv})=\left(2.37 \times 10^{-3} \mathrm{~L}\right)\left(3.41 \times 10^{-2} \mathrm{~s} / \mathrm{m}^{3}\right)\left(3.3 \times 10^{-4} \mathrm{~m}^{3} / \mathrm{s}\right)\left(6.1 \times 10^{3} \mathrm{~Sv} / \mathrm{L}\right)$

$D(S v)=1.6 \times 10^{-4} \mathrm{~Sv}$

244-AR Receiver Vault -

$D(\mathrm{SV})=\left(2.37 \times 10^{-3} \mathrm{~L}\right)\left(3.41 \times 10^{-2} \mathrm{~s} / \mathrm{m}^{3}\right)\left(3.3 \times 10^{-4} \mathrm{~m}^{3} / \mathrm{s}\right)\left(6.1 \times 10^{3} \mathrm{sv} / \mathrm{L}\right)$

$D(S V)=1.6 \times 10^{-4} \mathrm{SV}$

Compactor Building -

$D(\mathrm{~Sv})=\left(9.79 \times 10^{-4} \mathrm{~L}\right)\left(3.41 \times 10^{-2} \mathrm{~s} / \mathrm{m}^{3}\right)\left(3.3 \times 10^{-4} \mathrm{~m}^{3} / \mathrm{s}\right)\left(6.1 \times 10^{3} \mathrm{~Sv} / \mathrm{L}\right)$

$D(S v)=6.7 \times 10^{-5} \mathrm{~Sv}$

Catch Tanks -

$D(\mathrm{~Sv})=\left(9.79 \times 10^{-4} \mathrm{~L}\right)\left(3.41 \times 10^{-2} \mathrm{~s} / \mathrm{m}^{3}\right)\left(3.3 \times 10^{-4} \mathrm{~m}^{3} / \mathrm{s}\right)\left(6.1 \times 10^{3} \mathrm{~Sv} / \mathrm{L}\right)$

$D(S v)=6.7 \times 10^{-5} \mathrm{~Sv}$

Offsite Consequences:

$D(S v)=Q(L) \times \frac{X}{Q^{\prime}}\left(s / m^{3}\right) \times\left(\left(R\left[m^{3} / s\right] \times U L D_{H}[S v / L]\right)+U L D_{I}\left[S v-m^{3} / s-L\right]\right)$

SSTs with Active Ventilation -

$\begin{aligned} D(\mathrm{~Sv})= & \left(6.13 \times 10^{-3} \mathrm{~L}\right)\left(2.83 \times 10^{-5} \mathrm{~s} / \mathrm{m}^{3}\right)\left[\left(3.3 \times 10^{-4} \mathrm{~m}^{3} / \mathrm{s}\right)\left(2.2 \times 10^{5} \mathrm{sv} / \mathrm{L}\right)\right. \\ & \left.+4.15 \mathrm{~V}-\mathrm{m}^{3} / \mathrm{s}-\mathrm{L}\right]\end{aligned}$

$D(\mathrm{SV})=1.3 \times 10^{-5} \mathrm{SV}$

SSTs with Passive Ventilation 1 -

$\begin{aligned} D(\mathrm{~Sv})= & \left(1.27 \times 10^{-4} \mathrm{~L}\right)\left(2.83 \times 10^{-5} \mathrm{~s} / \mathrm{m}^{3}\right)\left[\left(3.3 \times 10^{-4} \mathrm{~m}^{3} / \mathrm{s}\right)\left(2.2 \times 10^{5} \mathrm{~Sv} / \mathrm{L}\right)\right. \\ & \left.+4.15 \mathrm{SV}-\mathrm{m}^{3} / \mathrm{s}-\mathrm{L}\right]\end{aligned}$

$D(S V)=2.8 \times 10^{-7} \mathrm{SV}$

SSTs with Passive Ventilation 2 -

$\begin{aligned} D(S v)= & \left(4.72 \times 10^{-5} \mathrm{~L}\right)\left(2.83 \times 10^{-5} \mathrm{~s} / \mathrm{m}^{3}\right)\left[\left(3.3 \times 10^{-4} \mathrm{~m}^{3} / \mathrm{s}\right)\left(2.2 \times 10^{5} \mathrm{~Sv} / \mathrm{L}\right)\right. \\ & \left.+4.1 \mathrm{~Sv}-\mathrm{m}^{3} / \mathrm{s}-\mathrm{L}\right]\end{aligned}$

$D(\mathrm{~Sv})=1.0 \times 10^{-7} \mathrm{~Sv}$ 


$$
\begin{aligned}
& \text { DCRTs - } \\
& D(S v)=\left(2.28 \times 10^{-3} \mathrm{~L}\right)\left(2.83 \times 10^{-5} \mathrm{~s} / \mathrm{m}^{3}\right)\left[\left(3.3 \times 10^{-4} \mathrm{~m}^{3} / \mathrm{s}\right)\left(1.1 \times 10^{4} \mathrm{~Sv} / \mathrm{L}\right)\right. \\
& \left.+0.052 \mathrm{~Sv}-\mathrm{m}^{3} / \mathrm{s}-\mathrm{L}\right] \\
& D(S v)=2.4 \times 10^{-7} \mathrm{~Sv} \\
& \text { DSTs - } \\
& \begin{aligned}
D(S v)= & \left(9.79 \times 10^{-4} \mathrm{~L}\right)\left(2.83 \times 10^{-5} \mathrm{~s} / \mathrm{m}^{3}\right)\left[\left(3.3 \times 10^{-4} \mathrm{~m}^{3} / \mathrm{s}\right)\left(6.1 \times 10^{3} \mathrm{~Sv} / \mathrm{L}\right)\right. \\
& \left.+0.068 \mathrm{~Sv}-\mathrm{m}^{3} / \mathrm{s}-\mathrm{L}\right]
\end{aligned} \\
& D(S v)=5.8 \times 10^{-8} \mathrm{~Sv}
\end{aligned}
$$

DSTs (using AWF Liquids) -

$\begin{aligned} D(S v)= & \left(5.62 \times 10^{-3} \mathrm{~L}\right)\left(2.83 \times 10^{-5} \mathrm{~s} / \mathrm{m}^{3}\right)\left[\left(3.3 \times 10^{-4} \mathrm{~m}^{3} / \mathrm{s}\right)\left(1.4 \times 10^{3} \mathrm{~Sv} / \mathrm{L}\right)\right. \\ & \left.+0.092 \mathrm{~Sv}-\mathrm{m}^{3} / \mathrm{s}-\mathrm{L}\right]\end{aligned}$

$D(S v)=8.8 \times 10^{-8} \mathrm{SV}$

204-AR Waste Unloading Facility -

$\begin{aligned} D(\mathrm{~Sv})= & \left(2.37 \times 10^{-3} \mathrm{~L}\right)\left(2.83 \times 10^{-5} \mathrm{~s} / \mathrm{m}^{3}\right)\left[\left(3.3 \times 10^{-4} \mathrm{~m}^{3} / \mathrm{s}\right)\left(6.1 \times 10^{3} \mathrm{~Sv} / \mathrm{L}\right)\right. \\ & \left.+0.068 \mathrm{~Sv}-\mathrm{m}^{3} / \mathrm{s}-\mathrm{L}\right]\end{aligned}$

$\mathrm{D}(\mathrm{Sv})=1.4 \times 10^{-7} \mathrm{~Sv}$

244-AR Receiver Vault -

$\begin{aligned} D(S v)= & \left(2.37 \times 10^{-3} \mathrm{~L}\right)\left(2.83 \times 10^{-5} \mathrm{~s} / \mathrm{m}^{3}\right)\left[\left(3.3 \times 10^{-4} \mathrm{~m}^{3} / \mathrm{s}\right)\left(6.1 \times 10^{3} \mathrm{~Sv} / \mathrm{L}\right)\right. \\ & \left.+0.068 \mathrm{~Sv}-\mathrm{m}^{3} / \mathrm{s}-\mathrm{L}\right]\end{aligned}$

$D(S v)=1.4 \times 10^{-7} \mathrm{SV}$

Compactor Building -

$\begin{aligned} D(S v)= & \left(9.79 \times 10^{-4} \mathrm{~L}\right)\left(2.83 \times 10^{-5} \mathrm{~s} / \mathrm{m}^{3}\right)\left[\left(3.3 \times 10^{-4} \mathrm{~m}^{3} / \mathrm{s}\right)\left(6.1 \times 10^{3} \mathrm{~Sv} / \mathrm{L}\right)\right. \\ & \left.+0.0685 \mathrm{~Sv}-\mathrm{m}^{3} / \mathrm{s}-\mathrm{L}\right]\end{aligned}$

$D(S v)=5.8 \times 10^{-8} \mathrm{~Sv}$

Catch Tanks -
$D(S v)=$
$\left(9.79 \times 10^{-4} \mathrm{~L}\right)\left(2.83 \times 10^{-5} \mathrm{~s} / \mathrm{m}^{3}\right)\left[\left(3.3 \times 10^{-4} \mathrm{~m}^{3} / \mathrm{s}\right)\left(6.1 \times 10^{3} \mathrm{~Sv} / \mathrm{L}\right)\right.$ $\left.+0.068 \mathrm{~Sv}-\mathrm{m}^{3} / \mathrm{s}-\mathrm{L}\right]$
$D(S v)=$
$5.8 \times 10^{-8} \mathrm{~Sv}$ 


\subsection{TOXICOLOGICAL EXPOSURE CONSEQUENCE ANALYSIS}

The following equation is used to calculate the peak concentration for a puff-type release from the release of HEPA filter contents as a result of a pressurization event:

\section{Toxicological Exposure Consequences (D) $=Q \cdot$ SOF}

Where $Q$ is equivalent to the amount ( $L$ ) shown in Table 1 for each TWRS ventilation system and,

SOF is the sum-of-fraction (1/L) as defined in Table 3-8 of WHC-SD-WM-SARR-011, Rev. 2 for the different waste types and is a function of accident frequency.

The toxicological exposure consequence values are calculated for the anticipated frequency category as follows.

\section{Onsite Exposure Consequences (for an Accident Frequency of Anticipated):}

SSTs with Active Ventilation -

$D=\left(6.13 \times 10^{-3} L\right)\left(1.2 \times 10^{4} 1 / L\right)$

$D=7.4 \times 10^{1}$

SSTs with Passive Ventilation 1 -

$D=\left(1.27 \times 10^{-4} \mathrm{~L}\right)\left(1.2 \times 10^{4} \mathrm{l} / \mathrm{L}\right)$

$D=1.5$

SSTs with Passive Ventilation 2 -

$D=\left(4.72 \times 10^{-5} \mathrm{~L}\right)\left(1.2 \times 10^{4} \mathrm{l} / \mathrm{L}\right)$

$D=5.6 \times 10^{-1}$

DCRTs -

$D=\left(2.28 \times 10^{-3} \mathrm{~L}\right)\left(2.8 \times 10^{3} \mathrm{l} / \mathrm{L}\right)$

$D=6.4$

DSTS -

$D=\left(9.79 \times 10^{-4} \mathrm{~L}\right)\left(2.9 \times 10^{3} \mathrm{l} / \mathrm{L}\right)$

$D=2.8$ 
DSTs (using AWF Liquids) -

$D=\left(5.62 \times 10^{-3} \mathrm{~L}\right)\left(2.9 \times 10^{3} \mathrm{l} / \mathrm{L}\right)$

$D=1.6 \times 10^{1}$

204-AR Waste Unloading Facility -

$D=\left(2.37 \times 10^{-3} \mathrm{~L}\right)\left(2.9 \times 10^{3} \mathrm{l} / \mathrm{L}\right)$

$D=6.9$

244-AR Receiver Vault -

$D=\left(2.37 \times 10^{-3} \mathrm{~L}\right)\left(2.9 \times 10^{3} \mathrm{~L} / \mathrm{L}\right)$

$D=6.9$

Compactor Building -

$D=\left(9.79 \times 10^{-4} \mathrm{~L}\right)\left(2.9 \times 10^{3} \mathrm{l} / \mathrm{L}\right)$

$D=2.8$

Catch Tanks -

$D=\left(9.79 \times 10^{-4} \mathrm{~L}\right)\left(2.9 \times 10^{3} \mathrm{l} / \mathrm{L}\right)$

$D=2.8$

Offsite Exposure Consequences (for an Accident Frequency of Anticipated): SSTs with Active Ventilation -

$D=\left(6.13 \times 10^{-3} \mathrm{~L}\right)\left(3.8 \times 10^{-1} \mathrm{l} / \mathrm{L}\right)$

$D=2.3 \times 10^{-3}$

SSTs with Passive Ventilation 1 -

$D=\left(1.27 \times 10^{-4} \mathrm{~L}\right)\left(3.8 \times 10^{-1} \mathrm{l} / \mathrm{L}\right)$

$D=4.8 \times 10^{-5}$

SSTs with Passive Ventilation 2 -

$D=\left(4.72 \times 10^{-5} \mathrm{~L}\right)\left(3.8 \times 10^{-1} \mathrm{l} / \mathrm{L}\right)$

$D=1.8 \times 10^{-5}$ 


$$
\begin{aligned}
& \text { DCRTs - } \\
& D=\left(2.28 \times 10^{-3} \mathrm{~L}\right)\left(3.2 \times 10^{-2} \mathrm{l} / \mathrm{L}\right) \\
& D=7.3 \times 10^{-5} \\
& \text { DSTs - } \\
& D=\left(9.79 \times 10^{-4} \mathrm{~L}\right)\left(3.4 \times 10^{-2} \mathrm{I} / \mathrm{L}\right) \\
& D=3.3 \times 10^{-5} \\
& \text { DSTs (using AWF Liquids) } \\
& D=\left(5.62 \times 10^{-3} \mathrm{~L}\right)\left(3.4 \times 10^{-2} \mathrm{I} / \mathrm{L}\right) \\
& D=1.9 \times 10^{-4}
\end{aligned}
$$

204-AR Waste UnToading Facility -

$D=\left(2.37 \times 10^{-3} \mathrm{~L}\right)\left(3.4 \times 10^{-2} \mathrm{l} / \mathrm{L}\right)$

$D=8.1 \times 10^{-5}$

244-AR Receiver Vault -

$D=\left(2.37 \times 10^{-3} \mathrm{~L}\right)\left(3.4 \times 10^{-2} \mathrm{l} / \mathrm{L}\right)$

$D=8.1 \times 10^{-5}$

\section{Compactor Building -}

$$
\begin{aligned}
& D=\left(9.79 \times 10^{-4} \mathrm{~L}\right)\left(3.4 \times 10^{-2} \mathrm{l} / \mathrm{L}\right) \\
& D=3.3 \times 10^{-5} \\
& \text { Catch Tanks }- \\
& D=\left(9.79 \times 10^{-4} \mathrm{~L}\right)\left(3.4 \times 10^{-2} \mathrm{l} / \mathrm{L}\right) \\
& D=3.3 \times 10^{-5}
\end{aligned}
$$

\subsection{ANALYSIS RESULTS}

The results of this analysis is summarized in Tables 5 through 8 . 
Table 5. Total Unmitigated Radiological Dose Consequences from a Pressurized HEPA Release Event and a Continuous, Unfiltered Release (2 sheets).

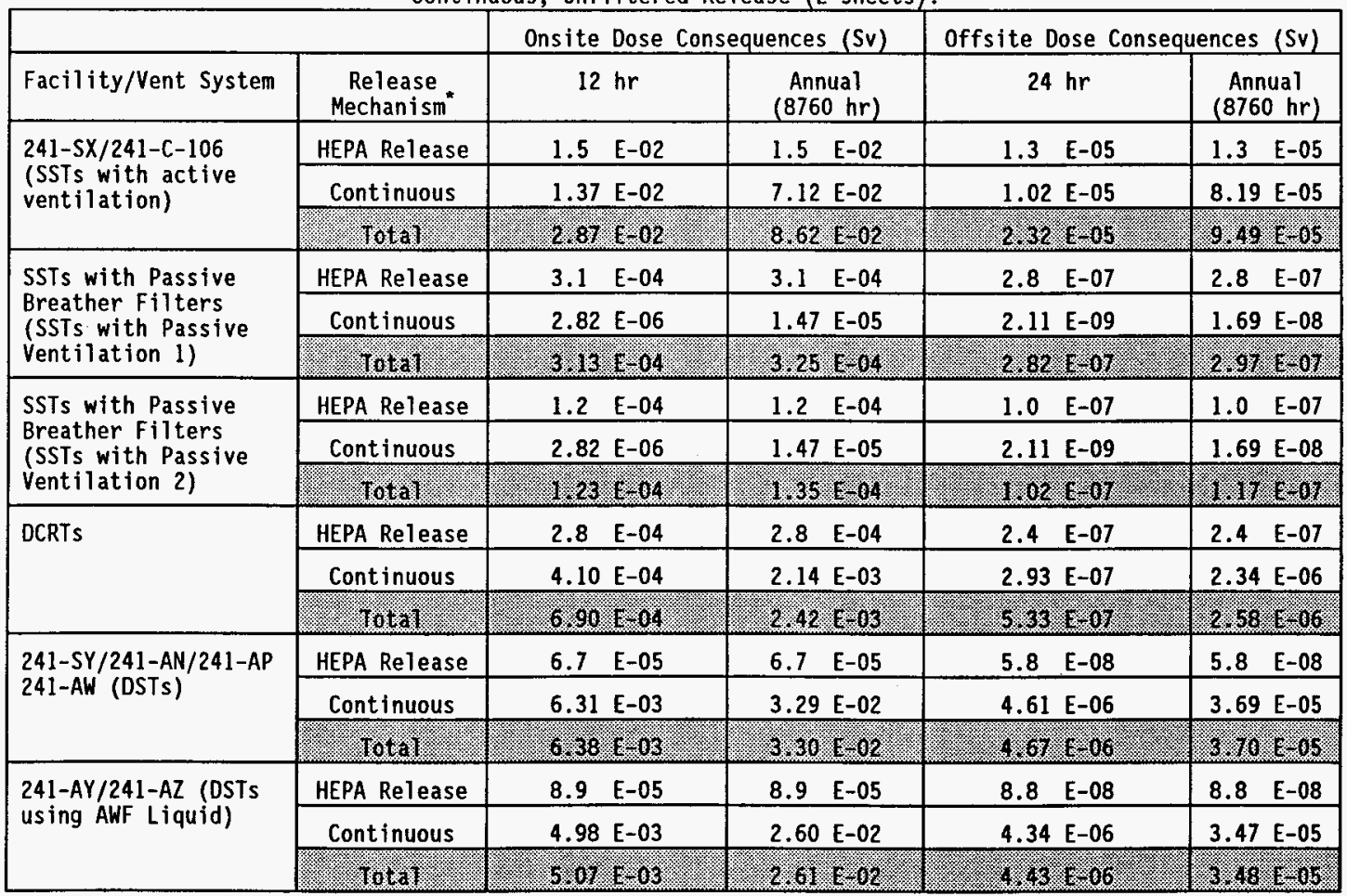


Table 5. Total Unmitigated Radiological Dose Consequences from a Pressurized HEPA Release Event and a Continuous, Unfiltered Release (2 sheets).

\begin{tabular}{|c|c|c|c|c|c|}
\hline \multirow[b]{2}{*}{ FaciTity/Vent System } & \multirow[b]{2}{*}{$\begin{array}{c}\text { Release } \\
\text { Mechanism }\end{array}$} & \multicolumn{2}{|c|}{ Onsite Dose Consequences (Sv) } & \multicolumn{2}{|c|}{ Offsite Dose Consequences (Sv) } \\
\hline & & $12 \mathrm{hr}$ & $\begin{array}{c}\text { Annual } \\
(8760 \mathrm{hr})\end{array}$ & $24 \mathrm{hr}$ & $\begin{array}{c}\text { Annual } \\
(8760 \mathrm{hr})\end{array}$ \\
\hline \multirow{3}{*}{$\begin{array}{l}\text { 204-AR Waste } \\
\text { Unloading Facility }\end{array}$} & HEPA Release & $1.6 \quad E-04$ & $1.6 \quad E-04$ & $1.4 \quad E-07$ & $1.4 \quad E-07$ \\
\hline & Continuous & $1.08 \mathrm{E}-04$ & $5.65 \mathrm{E}-04$ & $7.92 \mathrm{E}-08$ & $6.33 \mathrm{E}-07$ \\
\hline & (3. 100 tat & 2.68 .1 .04 & $1,25.1 .04$ & $2.19 .1=07$ & $7.7 .6 \cdot 1.09$ \\
\hline \multirow{3}{*}{$\begin{array}{l}\text { 244-AR Receiver } \\
\text { Vault }\end{array}$} & HEPA Release & $1.6 \quad \mathrm{E}-04$ & $1.6 \quad \mathrm{E}-04$ & $1.4 \quad E-07$ & $1.4 \quad E-07$ \\
\hline & Continuous & $8.13 E-04$ & $4.24 E-01$ & $5.94 E-07$ & $4.75 \mathrm{E}-06$ \\
\hline & 10 tal & $9.70 .2-04$ & $4.24 .8 .01 \cdot=$ & $7.34: 1.8 \%$ & $4.89 .6-06$ \\
\hline \multirow[t]{3}{*}{ Compactor Building } & HEPA Release & $6.7 \quad E-05$ & $6.7 \mathrm{E}-05$ & $5.8 \quad \mathrm{E}-08$ & $5.8 \quad E-08$ \\
\hline & Continuous & $N / A$ & $N / A$ & $N / A$ & N/A \\
\hline & $101 \mathrm{at}$ & $6.7=0.03$ & 6.2 .1 .05 & $58.6-2=08$ & $5.8 \cdot 2.08$ \\
\hline \multirow[t]{3}{*}{ Catch Tanks } & HEPA Release & $6.7 \mathrm{E}-05$ & $6.7 \quad \mathrm{E}-05$ & $5.8 \quad E-08$ & $5.8 \quad E-08$ \\
\hline & Continuous & $N / A$ & $N / A$ & $N / A$ & $N / A$ \\
\hline & lotal & 6.1 .1 .05 & $6.7 \div \cdot 1.05$. & $5.8 \cdot 1.08$ & $5.8 \cdot .1 .08$ \\
\hline
\end{tabular}

- The HEPA Release values are calculated in Section 5.0 and the Continuous values are taken from Table 3. rem $=100 \times S V$ 
Table 6. Total Unmitigated Toxicological Exposure Consequences from a Pressurized HEPA Release Event and a Continuous, Unfiltered Release for an Accident Frequency of Anticipated (2 sheets).

\begin{tabular}{|c|c|c|c|}
\hline Facility/Vent System & $\begin{array}{l}\text { Release } \\
\text { Mechanism }\end{array}$ & $\begin{array}{c}\text { Onsite } \\
\text { Exposure } \\
\text { Consequences }\end{array}$ & $\begin{array}{c}\text { Offsite } \\
\text { Exposure } \\
\text { Consequences } \\
\end{array}$ \\
\hline \multirow{3}{*}{$\begin{array}{l}241-S X / 241-C-106 \\
\text { (SSTs with active } \\
\text { ventilation) }\end{array}$} & HEPA Release & $7.4 \quad E+01$ & $2.3 \quad \mathrm{E}-03$ \\
\hline & Continuous & $1.32 \mathrm{E}-02$ & $3.10 \mathrm{E}-05$ \\
\hline & (2) & $1 \times 40 \cdot 6+1$ & 2. \\
\hline \multirow{3}{*}{$\begin{array}{l}\text { SSTs with Passive } \\
\text { Breather Filters (SSTs } \\
\text { with Passive } \\
\text { Ventilation 1) }\end{array}$} & HEPA Release & $1.5 E+00$ & $4.8 \quad E-05$ \\
\hline & Continuous ${ }^{1}$ & $1.43 \mathrm{E}-07$ & $2.24 \mathrm{E}-09$ \\
\hline & 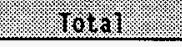 & $1 \times 30=400$ & $480 \cdot 6 \cdot 05$ \\
\hline \multirow{3}{*}{$\begin{array}{l}\text { SSTs with Passive } \\
\text { Breather Filters (SSTs } \\
\text { with Passive } \\
\text { Ventilation 2) }\end{array}$} & HEPA Release & $5.6 \quad \mathrm{E}-01$ & $1.8 \quad E-05$ \\
\hline & Continuous ${ }^{1}$ & 1.43 E-07 & $2.24 E-09$ \\
\hline & 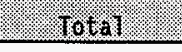 & 5.60 .500 & 1.80 .8 .05 \\
\hline \multirow[t]{3}{*}{ DCRTs } & HEPA Release & $6.4 \quad E+00$ & $E-05$ \\
\hline & Continuous & $1.90 E-03$ & $1.58 E-06$ \\
\hline & 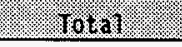 & 6.40 .600 & 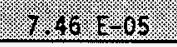 \\
\hline \multirow{3}{*}{$\begin{array}{l}241-S Y / 241-A N / 241-A P \\
241-A W(D S T S \text { ) }\end{array}$} & HEPA Release & $2.8 \quad E+00$ & $3.3 \quad \mathrm{E}-05$ \\
\hline & Continuous & $5.28 \quad E-02$ & $4.40 E-05$ \\
\hline & 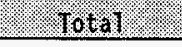 & 285.1600 & 17.20 .905 \\
\hline \multirow{3}{*}{$\begin{array}{l}\text { 241-AY/241-AZ (DSTs } \\
\text { using AWF Liquid) }\end{array}$} & HEPA Release & $1.6 \quad E+01$ & $1.9 \quad \mathrm{E}-04$ \\
\hline & Continuous & $1.89 \mathrm{E}-01$ & $1.59 \mathrm{E}-04$ \\
\hline & 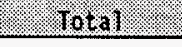 & $162 \times 101$ & (1) 89.6 .04 \\
\hline \multirow{3}{*}{$\begin{array}{l}\text { 204-AR Waste Unloading } \\
\text { Facility }\end{array}$} & HEPA Release & $6.9 \quad E+00$ & $8.1 \quad E-05$ \\
\hline & Continuous & $9.44 \mathrm{E}-04$ & $7.93 E-07$ \\
\hline & . & $6.906+00$ & $8 \times 18 \mathrm{c}=05$ \\
\hline \multirow[t]{3}{*}{ 244-AR Receiver Vault } & HEPA Release & $6.9 \quad E+00$ & $8.1 \quad E-05$ \\
\hline & Continuous & $6.80 \mathrm{E}-03$ & $5.66 E-06$ \\
\hline & $104 a t$. & $6 ., 90 \div 2.00$ & 6.67 .10 .05 \\
\hline \multirow[t]{3}{*}{ Compactor Building } & HEPA Release & $2.8 \quad E+00$ & $3.3 \quad E-05$ \\
\hline & Continuous & $N / A$ & $N / A$ \\
\hline & $2.1 \% 104 \times 1 \%$ & 2.8 .1800 & 3.3 .605 \\
\hline
\end{tabular}


Table 6. Total Unmitigated Toxicological Exposure Consequences from a Pressurized HEPA Release Event and a Continuous, Unfiltered Release for an Accident Frequency of Anticipated (2 sheets).

\begin{tabular}{|c|c|c|c|}
\hline Facility/Vent System & $\begin{array}{c}\text { Release } \\
\text { Mechanism }\end{array}$ & $\begin{array}{c}\text { Onsite } \\
\text { Exposure } \\
\text { Consequences } \\
\end{array}$ & $\begin{array}{c}\text { Offsite } \\
\text { Exposure } \\
\text { Consequences }\end{array}$ \\
\hline \multirow[t]{3}{*}{ Catch Tanks } & HEPA Release & $2.8 E+00$ & $3.3 \quad E-05$ \\
\hline & Continuous & $N / A$ & N/A \\
\hline & (10tat & Exoo & 200 \\
\hline
\end{tabular}

- The HEPA Release values are calculated in Section 6.0 and the Continuous values are taken from Table 4.

1 The continuous toxicological consequence values, as shown here are calculated in WHC-SD-WM-CN-062, using a frequency of unlikely.

rem $=100 \times S v$

The risk guidelines for radiological dose and toxicological exposure consequences are provided in Table 7 .

Table 7. Risk Guidelines for Radiological Dose and Toxicological Exposure Consequences.

Unmitigated Accident Frequencies:

Anticipated for SSTs with Active Ventilation, DCRTs, DSTs (including AWF), 204-AR Waste Unloading Facility, 244-AR Receiver Vault, and the Compactor Building.

Unlikely for SSTs with Passive Breather Filters (SSTs with Passive Ventilation 1 and 2 ), and catch tanks.

Total Consequences: See Tables 5 and 6 .

\begin{tabular}{|l|c|c|c|c|}
\hline \multirow{2}{*}{$\begin{array}{l}\text { Receptor/ } \\
\text { Hazard }\end{array}$} & \multirow{2}{*}{$\begin{array}{l}\text { Caiculated } \\
\text { Dose/Exposure }\end{array}$} & Anticipated & Unlikely & $\begin{array}{c}\text { Extremely } \\
\text { Unlikely }\end{array}$ \\
\cline { 3 - 5 } & See Table 5. & 1.0 E-03 Sv & 5.0 E-03 Sv & 4.0 E-02 Sv \\
Radiological & See Table 5. & 5.0 E-03 Sv & 5.0 E-02 Sv & 1.0 E-01 Sv \\
\hline $\begin{array}{l}\text { Onsite/ } \\
\text { Radiological }\end{array}$ & See Table 6. & 1 & 1 & 1 \\
\hline $\begin{array}{l}\text { Offsite/ } \\
\text { Toxicological }\end{array}$ & See Table 6. & 1 & 1 & 1 \\
\hline $\begin{array}{l}\text { Onsite/ } \\
\text { Toxicological }\end{array}$ & & & & \\
\hline
\end{tabular}




\subsection{CONCLUSIONS}

The total unmitigated radiological consequences from unfiltered releases due to pressurization for single-shell tanks with active ventilation, double-shell tanks (including AWF tanks), and the 244-AR receiver vault as shown in Table 5 are above the risk evaluation guidelines for an anticipated event $\left(5.0 \times 10^{-3} \mathrm{~Sv}\right)$. Total unmitigated radiological doses to the offsite receptor are below the risk guidelines in all cases.

The total unmitigated toxicological exposure consequences from unfiltered releases due to pressurization exceed the onsite risk guidelines in all cases except for the single-shell tanks with passive breather filters (SSTs with passive ventilation 1 and 2 ) as shown in Table 6 . Total unmitigated toxicological exposure consequences to the offsite receptor are below the risk guidelines in all cases.

Controls to either mitigate or prevent the risk associated with accident scenario are not developed or proposed in this document. This is due to the fact, as discussed in Section 1.0, that a pressurization does not occur independent of an additional accident initiator. The appropriate controls are left to the specific accident scenario that may include a tank headspace pressurization resulting in a release similar to the ones calculated here.

\subsection{REFERENCES}

DOE-HDBK-3010-94, 1994, DOE Handbook Airborne Release Fractions/Rates and Restorab7e Fraction for Nonreactor Nuclear Facilities, U.S. Department of Energy, Richl and, Washington.

WHC-SD-WM-SARR-011, Rev. 2, Toxicological Chemical Considerations for Tank Farm Releases, Westinghouse Hanford Company, Richland, Washington.

WHC-SD-WM-SARR-016, Rev. 2, Tank Waste Compositions and Atmospheric Dispersion Coefficients for use in ASA Consequence Assessments, Westinghouse Hanford Company, Richland, Washington.

WHC-SD-WM-SARR-037, Rev. 0, Development of Radiological Concentrations and Unit Liter Doses for TWRS FSAR Radiological Consequence Calculations, Westinghouse Hanford Company, Richland, Washington.

WHC-SD-WM-CN-054, Rev. 0, Waste Tank Ventilation System Waste Material Accumulations, Westinghouse Hanford Company, Richland, Washington.

WHC-SD-WM-CN-062, Rev. 0, HEPA Fi7ter Fire (and Subsequent Unfiltered Release), Westinghouse Hanford Company, Richland, Washington.

WHC-CM-4-46, Rev. 1, Safety Analysis Manual, Section 4.0, Westinghouse Hanford Company, Richland, Washington. 
WHC-SD-WM-CN-063 REV 0

APPENDIX A

PEER REVIEW AND HEDOP REVIEW CHECKLISTS 
WHC-SD-WM-CN-063 REV 0

This page intentionally left blank. 


\section{CHECKLIST FOR PEER REVIEW}

Document Reviewed: WHC-SD-WM-CN-063, Rev. 0, Unfiltered HEPA Filter Release Due to Pressurization

Scope of Review: Entire document.

Yes No NA

[ ] [ ] $*$ Previous reviews complete and cover analysis, up to scope of

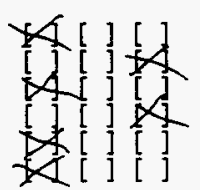

[ ] [ ] $1 \%$

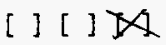

$X[1][1]$

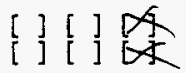

$\pm[1][]$

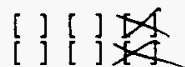

$\mathbb{X}[\mathrm{X}[\mathrm{c}]$

[]$^{\prime}[] \not L$

[ ] [ $\mathcal{K}^{*}$ Review ca]culations, comments, and/or notes are attached.
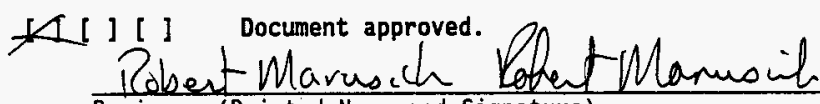

Reviewer (Printed Name and Signature)

* Any calculations, comments, or notes generated as part of this review should be signed, dated and attached to this checklist. Such material should be labeled and recorded in such a manner as to be intelligible to a technically qualified third party. 


\section{WHC-SD-WM-CN-063 REV 0 \\ HEDOP REVIEN CHECKLIST \\ for \\ Radiological and Nonradiological Release Calculations}

Document reviewed (include title or description of calculation, document number, author, and date, as applicable):

WHC-SD-WM-CN-063, Rev. 0, Unfiltered HEPA Fi7ter Release Due to Pressurization

Submitted by: Grant W. Ryan Date Submitted: 8/15/96

Scope of Review: Radiological Dose and Toxicological Exposure Calculations.

YES NO* N/A

[4 [ ] [ ] 1. A detailed technica] review and approval of the environmental transport and dose calculation portion of the analysis has been performed and documented.

$\infty$ [ ] [ ] 2. Detailed technical review(s) and approval(s) of scenario and release determinations have been performed and documented.

[ ] [ ] 3. HEDOP-approved code(s) were used.

[ ] [ ] [4 4. Receptor ]ocations were selected according to HEDOP recommendations.

\&] [ ] [ ] 5. All applicable environmental pathways and code options

DA [ ] [ ] 6. Hanford site data were used.

[ ] [ ]. 7. Model adjustments external to the computer program were justified and performed correctly.

14 [ ] [ ] 8. The analysis is consistent with HEDOP recommendations.

9 . Supporting notes, calculations, comments, comment resolutions, or other information is attached. (Use the "Page $I$ of $X "$ page numbering format and sign and date each added page.)

$\mathbb{M}[\mathrm{I}$

10. Approval is granted on behalf of the Hanford Environmental Dose Overview Panel.

- A11 "NO" responses must be explained and use of nonstandard methods justified.

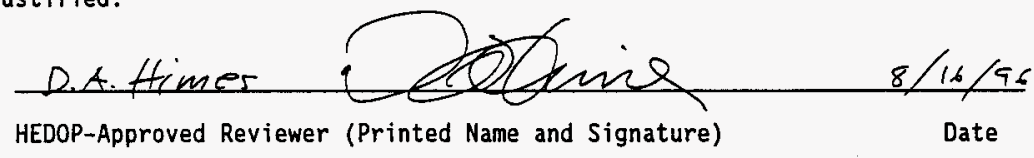

COMMENTS (add additional signed and dated pages if necessary): 


\begin{tabular}{|c|c|c|c|c|c|}
\hline \multicolumn{6}{|c|}{ DISTRIBUTION SHEET } \\
\hline \multirow{2}{*}{$\begin{array}{l}\text { To } \\
\text { Distribution }\end{array}$} & \multirow{2}{*}{\multicolumn{3}{|c|}{$\begin{array}{l}\text { From } \\
\text { G. W. Ryan }\end{array}$}} & \multicolumn{2}{|l|}{ Page 1 of 1} \\
\hline & & & & \multicolumn{2}{|c|}{ Date $8 / 16 / 96$} \\
\hline \multicolumn{4}{|c|}{ Project Title/Work Order } & \multicolumn{2}{|c|}{ EDT No. 614552} \\
\hline \multicolumn{4}{|c|}{$\begin{array}{l}\text { Releases From Failed HEPA Filters Due to an Overpressurization } \\
\text { Event, WHC-SD-WM-CN-063, Rev. } 0\end{array}$} & \multicolumn{2}{|c|}{ ECN No. N/A } \\
\hline Name & MSIN & $\begin{array}{c}\text { Text } \\
\text { With All } \\
\text { Attach. }\end{array}$ & Text Only & $\begin{array}{l}\text { Attach./ } \\
\text { Appendix } \\
\text { Only }\end{array}$ & $\begin{array}{l}\text { EDT/ECN } \\
\text { Only }\end{array}$ \\
\hline $\begin{array}{l}\text { E. R. Bruschi } \\
\text { C. Carro } \\
\text { D. S. Leach } \\
\text { TWRS S \& L Project Files ( } 6) \\
\text { G. W. Ryan (3) } \\
\text { Central Files (Original + } 1 \text { ) }\end{array}$ & $\begin{array}{l}\text { A2-34 } \\
\text { A2-34 } \\
\text { A3-34 } \\
\text { A2-26 } \\
\text { A3-37 } \\
\text { A3-88 }\end{array}$ & $\begin{array}{l}x \\
x \\
x \\
x \\
x \\
x\end{array}$ & & & \\
\hline
\end{tabular}

